PROCEEDINGS OF THE

AMERICAN MATHEMATICAL SOCIETY

Volume 132, Number 7 , Pages 2039-2049

S 0002-9939(04)07380-0

Article electronically published on February 19, 2004

\title{
GEOMETRY OF EPIMORPHISMS AND FRAMES
}

\author{
GUSTAVO CORACH, MIRIAM PACHECO, AND DEMETRIO STOJANOFF \\ (Communicated by David R. Larson) \\ Dedicated to our friend Jorge Solomin
}

\begin{abstract}
Using a bijection between the set $\mathcal{B}_{\mathcal{H}}$ of all Bessel sequences in a (separable) Hilbert space $\mathcal{H}$ and the space $L\left(\ell^{2}, \mathcal{H}\right)$ of all (bounded linear) operators from $\ell^{2}$ to $\mathcal{H}$, we endow the set $\mathcal{F}$ of all frames in $\mathcal{H}$ with a natural topology for which we determine the connected components of $\mathcal{F}$. We show that each component is a homogeneous space of the group $G L\left(\ell^{2}\right)$ of invertible operators of $\ell^{2}$. This geometrical result shows that every smooth curve in $\mathcal{F}$ can be lifted to a curve in $G L\left(\ell^{2}\right)$ : given a smooth curve $\gamma$ in $\mathcal{F}$ such that $\gamma(0)=\Xi$, there exists a smooth curve $\Gamma$ in $G L\left(\ell^{2}\right)$ such that $\gamma=\Gamma \cdot \Xi$, where the dot indicates the action of $G L\left(\ell^{2}\right)$ over $\mathcal{F}$. We also present a similar study of the set of Riesz sequences.
\end{abstract}

\section{INTRODUCTION}

Let $\mathcal{H}$ be a (complex, separable) Hilbert space, $L\left(\ell^{2}, \mathcal{H}\right)$ the algebra of all bounded linear operators from $\ell^{2}$ to $\mathcal{H}$ and $\mathcal{E}$ the subset of $L\left(\ell^{2}, \mathcal{H}\right)$ consisting of all epimorphisms from $\ell^{2}$ onto $\mathcal{H}$.

A sequence $\left\{\xi_{n}\right\}$ of elements of $\mathcal{H}$ is called a frame if there exist positive constants $A, B$ such that

$$
A\|\xi\|^{2} \leq \sum\left|\left\langle\xi, \xi_{n}\right\rangle\right|^{2} \leq B\|\xi\|^{2}
$$

for all $\xi \in \mathcal{H}$. Denote by $\mathcal{F}$ the set of all frames in $\mathcal{H}$. Frames have been introduced by Duffin and Schaeffer in [13], in connection with nonharmonic Fourier series, but they attracted more attention since the begining of wavelet theory due, in particular, to the fundamental paper [11]. The reader will find many relevant results and facts on frame theory in the book 10 by I. Daubechies, and in several papers, in particular the survey by C. Heil and D. Walnut [15], the monograph [14] by D. Han and D. Larson, the exposition [4] of P. Casazza and the survey [5] by O. Christensen. The papers [16 by J. R. Holub and 11 by A. Aldroubi contain some results related to ours. Also, in [2, R. Balan introduces a decomposition in $\mathcal{F}$ and defines a metric on each "component" of the partition. In this paper, we proceed in a different way by defining a natural topology in the set $\mathcal{F}$ of all frames $\Xi=\left\{\xi_{n}\right\}$ in $\mathcal{H}$. We characterize the connected components of $\mathcal{F}$ and show that each component

Received by the editors October 28, 2002 and, in revised form, March 19, 2003.

2000 Mathematics Subject Classification. Primary 42C15, 47B99, 58B10.

Key words and phrases. Fibre bundle, frame, epimorphisms, Bessel sequence, Riesz sequence. Partially supported by ANPCYT (03-9521), UBACYT (X050) and UNLP (11 X350). 
is a homogeneous space of the group $G L\left(\ell^{2}\right)$ of all invertible operators on $\ell^{2}$. These facts come from the existence of a natural action of $G L\left(\ell^{2}\right)$ over $\mathcal{F}$. We get all these results in an indirect way. In fact, we first study the topology of the set $\mathcal{E}$ of all (bounded linear) epimorphisms $\ell^{2} \rightarrow \mathcal{H}$ and define an action $G L\left(\ell^{2}\right) \times \mathcal{E} \rightarrow \mathcal{E}$ to characterize the connected components of $\mathcal{E}$. Then, we define a Banach space $\mathcal{B}_{\mathcal{H}}$ of sequences in $\mathcal{H}$ ("Bessel sequences") and a natural isomorphism from $L\left(\ell^{2}, \mathcal{H}\right)$ onto $\mathcal{B}_{\mathcal{H}}$ that maps $\mathcal{E}$ onto $\mathcal{F}$. By means of this isomorphism all facts about $\mathcal{E}$ are translated to $\mathcal{F}$.

The paper is divided into two parts: in the first part we endow $\mathcal{E}$ with the topology induced by the operator norm in $L\left(\ell^{2}, \mathcal{H}\right) . \mathcal{E}$ is an open subset of $L\left(\ell^{2}, \mathcal{H}\right)$ and there is a natural action of $G L\left(\ell^{2}\right)$ over $\mathcal{E}$, by multiplication on the right. The orbits of this action are the connected components of $\mathcal{E}$. More precisely, for each $n \in \mathbb{N} \cup\{+\infty\}$ the set $\mathcal{E}_{n}=\{T \in \mathcal{E}: \operatorname{dim} \operatorname{ker} T=n\}$ is a connected component of $\mathcal{E}$ and, as an orbit of the action, it is a homogeneous space of $G L\left(\ell^{2}\right)$. As such, it has several pleasant geometric properties. In particular, continuous (resp. smooth) curves in $\mathcal{E}_{n}$ lift to continuous (resp. smooth) curves in $G L\left(\ell^{2}\right)$. Thus, any curve $\gamma$ in $\mathcal{E}_{n}$ has the form $\gamma(t)=T \cdot \Gamma(t)$ for some curve $\Gamma$ in $G L\left(\ell^{2}\right)$. In the second part of the paper, we observe that the bijection between $\mathcal{F}$ and $\mathcal{E}$ is the restriction of a natural bijection between $L\left(\ell^{2}, \mathcal{H}\right)$ and the space $\mathcal{B}_{\mathcal{H}}$ of all Bessel sequences in $\mathcal{H}$. It turns out that there is a natural Banach space structure on $\mathcal{B}_{\mathcal{H}}$ such that the bijection is an isomorphism of Banach spaces. Thus, the connected components of $\mathcal{F}$, which correspond bijectively with the connected components of $\mathcal{E}$, are easily determined, and the fibration properties of these components allow us to characterize their curves by mean of curves in $G L\left(\ell^{2}\right)$. Finally, using the facts that epimorphisms correspond bijectively with monomorphisms with closed range and that these operators correspond with the set $\mathcal{R}$ of Riesz sequences, we get a similar geometrical description of $\mathcal{R}$.

\section{Geometry of epimorphisms}

Throughout, $\mathcal{H}$ denotes a Hilbert space, $L(\mathcal{H})$ is the algebra of all linear bounded operators on $\mathcal{H}, L(\mathcal{H})^{+}$is the subset of $L(\mathcal{H})$ of all (selfadjoint) positive operators, $G L(\mathcal{H})$ is the group of all invertible operators in $L(\mathcal{H})$ and $G L(\mathcal{H})^{+}=G L(\mathcal{H}) \cap$ $L(\mathcal{H})^{+}$(positive invertible operators). For every $C \in L(\mathcal{H})$ its range is denoted by $R(C)$ and its nullspace by $\operatorname{ker} C$.

Consider two Hilbert spaces $\mathcal{H}$ and $\mathcal{K}$ and the space $L(\mathcal{H}, \mathcal{K})$ of all linear bounded operators from $\mathcal{H}$ to $\mathcal{K}$. We denote by $\mathcal{E}$ the set of all epimorphisms in $L(\mathcal{H}, \mathcal{K})$ :

$$
\mathcal{E}=\{T \in L(\mathcal{H}, \mathcal{K}): R(T)=\mathcal{K}\}
$$

An interesting subset of $\mathcal{E}$ is the space $\mathcal{E}^{o}$ of co-isometries:

$$
\mathcal{E}^{o}=\left\{T \in L(\mathcal{H}, \mathcal{K}): T T^{*}=I_{\mathcal{K}}\right\}
$$

The following result is elementary and will be used frequently:

Proposition 2.1. Let $T \in L(\mathcal{H}, \mathcal{K})$. Then the following properties are equivalent:

(1) $T \in \mathcal{E}$;

(2) $T^{*}$ is injective and $R\left(T^{*}\right)$ is closed (i.e., $T^{*}$ is bounded from below);

(3) there exists $S \in L(\mathcal{K}, \mathcal{H})$ such that $T S=I_{\mathcal{K}}$;

(4) $T T^{*} \in G L(\mathcal{K})$;

(5) $T A T^{*} \in G L(\mathcal{K})^{+}$for some (or any) $A \in G L(\mathcal{H})^{+}$; 
(6) the transformation $X \mapsto T X$ is an epimorphism from $L(\mathcal{H})$ onto $L(\mathcal{H}, \mathcal{K})$;

Remark 2.2. Recall the left polar decomposition of $T \in L(\mathcal{H}, \mathcal{K})$ : there exist $A \in$ $L(\mathcal{H})^{+}$and a partial isometry $V \in L(\mathcal{H}, \mathcal{K})$ such that $T=V A$. $A$ is uniquely determined by the equation $A=|T|=\left(T^{*} T\right)^{1 / 2}$, and $V$ is unique provided that $\operatorname{ker} V=\operatorname{ker} T$.

We also have the right polar decomposition of $T \in L(\mathcal{H}, \mathcal{K})$ : there exist $B \in$ $L(\mathcal{K})^{+}$and a partial isometry $W \in L(\mathcal{H}, \mathcal{K})$ such that $T=B W . B$ is uniquely determined by the equation $B=\left|T^{*}\right|=\left(T T^{*}\right)^{1 / 2}$, and $W$ is unique provided that $\operatorname{ker} W=\operatorname{ker} T$.

With these facts and notation, we can add two different equivalent conditions to the list of Proposition 2.1.

7. $B \in G L(\mathcal{K})$;

8. $W \in \mathcal{E}^{o}$ (i.e., $W$ is a co-isometry), and $R(T)$ is closed.

In the rest of this section, we only consider the topology induced on $L(\mathcal{H}, \mathcal{K})$ and its subsets by the operator norm.

Corollary 2.3. $\mathcal{E}$ is open in $L(\mathcal{H}, \mathcal{K})$, and $\mathcal{E}^{o}$ is closed in $\mathcal{E}$.

Proof. Consider the continuous map $\alpha: L(\mathcal{H}, \mathcal{K}) \rightarrow L(\mathcal{K})$ given by $\alpha(T)=T T^{*}$, $T \in L(\mathcal{H}, \mathcal{K})$. Then $\mathcal{E}=\alpha^{-1}(G L(\mathcal{H}))$ and $\mathcal{E}^{o}=\alpha^{-1}(\{I\})$.

Recall that, given a topological space $X$ and $A \subseteq X$, then $A$ is said to be a strong deformation retract of $X$ if there is a continuous map $\rho: X \times[0,1] \rightarrow X$ such that

(1) for all $x \in X, \rho(x, 0)=x$ and $\rho(x, 1) \in A$;

(2) for all $a \in A$ and $t \in[0,1], \rho(a, t)=a$.

The map $\rho$ is called a strong deformation retraction. In this case, $X$ and $A$ have the same homotopy groups (see, for example, [17]). The following observation will be used later.

Proposition 2.4. $\mathcal{E}^{o}$ is a strong deformation retract of $\mathcal{E}$.

Proof. Consider the map $\rho: \mathcal{E} \rightarrow \mathcal{E}^{o}$ given by $\rho(T)=\left(T T^{*}\right)^{-1 / 2} T$. Clearly $\rho$ is a continuous retraction. The map $\rho: \mathcal{E} \times[0,1] \rightarrow \mathcal{E}$ given by

$$
\rho(T, t)=\rho_{t}(T)=\left(T T^{*}\right)^{-t / 2} T, \quad(T, t) \in \mathcal{E} \times[0,1],
$$

defines a strong deformation retraction between $1_{\mathcal{E}}$ and $\rho$.

If $T \in \mathcal{E}^{o}$, then it is easy to see that $T T^{*}=I_{\mathcal{K}}$ and $T^{*} T=P_{(\operatorname{ker} T)^{\perp}}=P_{R\left(T^{*}\right)}$. As a consequence, we get that the map $\theta: \mathcal{E} \rightarrow L(\mathcal{H})$ given by $\theta(T)=P_{(\operatorname{ker} T)^{\perp}}$ is continuous. Recall that the Moore-Penrose pseudoinverse of a closed range operator $T \in L(\mathcal{H}, \mathcal{K})$ is the unique operator $T^{\dagger} \in L(\mathcal{K}, \mathcal{H})$ that satisfies $T T^{\dagger} T=T$, $T^{\dagger} T T^{\dagger}=T^{\dagger},\left(T T^{\dagger}\right)^{*}=T T^{\dagger}$ and $\left(T^{\dagger} T\right)^{*}=T^{\dagger} T$. The reader is referred to 12 or 18] for properties and applications of this notion. One of the properties of $T^{\dagger}$ that we need is the identity $T^{\dagger} T=P_{(\operatorname{ker} T)^{\perp}}$. From this fact and the continuity of $\theta$, we get:

Proposition 2.5. If $T \in \mathcal{E}$, the Moore-Penrose pseudoinverse of $T$ is

$$
T^{\dagger}=T^{*}\left(T T^{*}\right)^{-1} \quad \text { and } \quad P_{(\operatorname{ker} T)^{\perp}}=T^{*}\left(T T^{*}\right)^{-1} T .
$$


Proof. Let $X=T^{*}\left(T T^{*}\right)^{-1}$. Then $T X=I, X T X=X$ and $T X T=T$. On the other hand, $T X=I$ and $X T=T^{*}\left(T T^{*}\right)^{-1} T$ are both selfadjoint. Therefore, $X=T^{\dagger}$ and $P_{(\operatorname{ker} T)^{\perp}}=T^{\dagger} T=T^{*}\left(T T^{*}\right)^{-1} T$.

Corollary 2.6. The maps $\mu: \mathcal{E} \rightarrow L(\mathcal{K}, \mathcal{H})$ and $\theta: \mathcal{E} \rightarrow L(\mathcal{H})$ defined by $\mu(T)=T^{\dagger}$ and $\theta(T)=P_{(\operatorname{ker} T)^{\perp}}$, respectively, are real analytic.

The action of $G L(\mathcal{H})$ on $\mathcal{E}$. Consider the following left action of $G L(\mathcal{H})$ on $\mathcal{E}$ :

$$
G L(\mathcal{H}) \times \mathcal{E} \rightarrow \mathcal{E}, \quad(V, T) \mapsto T V^{-1} .
$$

The orbit of $T \in \mathcal{E}$ by this action is the set $T \cdot G L(\mathcal{H})$.

Theorem 2.7. Let $T \in \mathcal{E}$. Then the orbit $T \cdot G L(\mathcal{H})$ is open, and it is the connected component of $T$ in $\mathcal{E}$.

Proof. Since $G L(\mathcal{H})$ is open and connected in $L(\mathcal{H})$ and $X \mapsto T X$ is continuous and linear from $L(\mathcal{H})$ onto $L(\mathcal{H}, \mathcal{K})$, it follows that $T \cdot G L(\mathcal{H})$ is open (by the open mapping theorem) and connected in $L(\mathcal{H}, \mathcal{K})$.

For each $n \in \mathbb{N} \cup\{\infty\}$, define the sets

$$
\mathcal{E}_{n}=\{T \in \mathcal{E}: \operatorname{dim} \operatorname{ker} T=n\} \quad \text { and } \quad \mathcal{E}_{n}^{o}:=\left\{S \in \mathcal{E}^{o}: \operatorname{dim} \operatorname{ker} S=n\right\},
$$

so that

$$
\mathcal{E}=\bigcup_{n \in \mathbb{N} \cup\{\infty\}} \mathcal{E}_{n} \quad \text { and } \quad \mathcal{E}^{o}=\bigcup_{n \in \mathbb{N} \cup\{\infty\}} \mathcal{E}_{n}^{o} .
$$

We prove that the connected components of $\mathcal{E}$ (resp. $\mathcal{E}^{o}$ ) are, precisely, $\mathcal{E}_{n}$ (resp. $\left.\mathcal{E}_{n}^{o}\right)$.

Proposition 2.8. Let $n \in \mathbb{N} \cup\{\infty\}$. Then:

(1) Given $T_{1}, T_{2} \in \mathcal{E}_{n}$, there exists $V \in G L(\mathcal{H})$ such that $T_{2}=T_{1} V$. In other words, if $T \in \mathcal{E}_{n}$, then $\mathcal{E}_{n}=T \cdot G L(\mathcal{H})$.

(2) Given $T_{1}, T_{2} \in \mathcal{E}_{n}^{o}$, there exists $u \in \mathcal{U}(\mathcal{H})$ such that $T_{2}=T_{1} U$. In other words, if $T \in \mathcal{E}_{n}^{o}$, then $\mathcal{E}_{n}^{o}=T \cdot \mathcal{U}(\mathcal{H})=\left\{T U^{*}: U \in \mathcal{U}(\mathcal{H})\right\}$.

Proof. (1) The operator $V_{1}=T_{1}^{\dagger} T_{2}: \operatorname{ker} T_{2}^{\perp} \rightarrow \operatorname{ker} T_{1}^{\perp}$ is invertible. It can be "completed" to an invertible operator $V=V_{1}+V_{2} \in G L(\mathcal{H})$, choosing any isomorphism $V_{2}: \operatorname{ker} T_{2} \rightarrow \operatorname{ker} T_{1}$. It is clear that $T_{1} V=T_{1} V_{1}=T_{2}$.

(2) It follows the same lines, but $V_{1}$ and $V_{2}$ are unitaries.

Remark 2.9. As the referee pointed out to us, the connected components of the set of semi-Fredholm operators in $L(\mathcal{H}, \mathcal{K})$ are determined by the index (see Cordes and Labrousse [9). From this fact it can be easily deduced that the connected components of the set of all epimorphisms are determined by the nullity (i.e., dimension of the nullspace). The advantage of the present approach is that the connected components are characterized as the orbits of a natural action of $G L(\mathcal{H})$ over $\mathcal{E}$. Observe that, in order to get the complete result of Cordes and Labrousse by a similar method, one should define an action of a convenient group over the set of all semi-Fredholm operators in $L(\mathcal{H}, \mathcal{K})$. This approach is possible. We plan to do it elsewhere. 
Proposition 2.10. Let $T \in \mathcal{E}$. Then the mapping

$$
\tau_{T}: G L(\mathcal{H}) \rightarrow \mathcal{E}, \quad \tau_{T}(V)=T V^{-1}
$$

admits analytic local cross sections.

Proof. We must prove that for every $T \in \mathcal{E}$ there exists a neighborhood $\mathcal{B}$ of $T$ in $\mathcal{E}$ and an analytic map $\sigma: \mathcal{B} \rightarrow G L(\mathcal{H})$ such that $\tau_{T}\left(\sigma\left(T^{\prime}\right)\right)=T^{\prime}$ for all $T^{\prime} \in \mathcal{B}$. Choose $S \in L(\mathcal{K}, \mathcal{H})$ such that $T S=I_{\mathcal{K}}$. Taking $\varepsilon=\|S\|^{-1}$, if $W \in L(\mathcal{H}, \mathcal{K})$ and $\|T-W\|<\varepsilon$, then $\left\|I_{\mathcal{K}}-W S\right\| \leq\|T-W\|\|S\|<1$. Hence $W S \in G L(\mathcal{K})$ and $W \in \mathcal{E}$. Also, $S W+\left(I_{\mathcal{H}}-S T\right) \in G L(\mathcal{H})$, because $\left\|S W+\left(I_{\mathcal{H}}-S T\right)-I_{\mathcal{H}}\right\| \leq$ $\|S\|\|W-T\|<1$. For $W \in L(\mathcal{H}, \mathcal{K})$ such that $\|T-W\|<\varepsilon$, define

$$
\sigma(W)=\left(S W+\left(I_{\mathcal{H}}-S T\right)\right)^{-1} .
$$

Then the map $\sigma$ is analytic, and it is a local cross section of $\tau_{T}$, because $\tau_{T}(\sigma(W))=$ $T \sigma(W)^{-1}=T\left(S W+\left(I_{\mathcal{H}}-S T\right)\right)=W$.

Corollary 2.11. Let $T \in \mathcal{E}$ and $n=\operatorname{dim} \operatorname{ker} T$. Then $\mathcal{E}_{n}=T \cdot G L(\mathcal{H})$ is homeomorphic to the homogeneous space $G L(\mathcal{H}) / \mathcal{I}_{T}$, where $\mathcal{I}_{T}$ is the isotropy group at $T$ of the action of $G L(\mathcal{H})$ on $\mathcal{E}$, i.e.,

$$
\mathcal{I}_{T}=\{V \in G L(\mathcal{H}): T V=T\} .
$$

Proposition 2.12. Let $T \in \mathcal{E}$, and denote by $P=T^{\dagger} T=P_{\operatorname{ker} T \perp}$. Then the isotropy group $\mathcal{I}_{T}$ of $T$, defined in (2.1), can be characterized in the matrix representation of $L(\mathcal{H})$ given by $P$, by

$$
\mathcal{I}_{T}=\left\{\left(\begin{array}{ll}
1 & 0 \\
x & y
\end{array}\right): y \in G L(\operatorname{ker} T)\right\} .
$$

Proof. The matrix form of $T$ is $T=\left(\begin{array}{cc}T_{1} & 0 \\ T_{2} & 0\end{array}\right)$ with $T_{1}^{*} T_{1}+T_{2}^{*} T_{2} \in G L\left(\operatorname{ker} T^{\perp}\right)^{+}$. If $V=\left(\begin{array}{ll}a & b \\ x & y\end{array}\right) \in G L(\mathcal{H})$, then

$$
T V=T \Longleftrightarrow\left(\begin{array}{cc}
T_{1} a & T_{1} b \\
T_{2} a & T_{2} b
\end{array}\right)=T .
$$

This shows that if $a=1$ and $b=0$, then $V \in \mathcal{I}_{T}$. On the other hand, if $T V=T$, then by equation (2.3),

$$
T^{*} T=\left(\begin{array}{cc}
T_{1}^{*} T_{1}+T_{2}^{*} T_{2} & 0 \\
0 & 0
\end{array}\right)=\left(\begin{array}{cc}
a^{*}\left(T_{1}^{*} T_{1}+T_{2}^{*} T_{2}\right) a & * \\
* & b^{*}\left(T_{1}^{*} T_{1}+T_{2}^{*} T_{2}\right) b
\end{array}\right),
$$

showing that $a=1$ and $b=0$. Finally, the fact that $y \in G L(\operatorname{ker} T)$ is equivalent to the fact that $V \in G L(\mathcal{H})$.

Remark 2.13. Fix $n \in \mathbb{N} \cup\{\infty\}$. $\mathcal{E}_{n}$ has a natural structure of an analytic submanifold of $L(\mathcal{H}, \mathcal{K})$ as an open subset. By equation $(2.2), \mathcal{I}_{T}$ is a regular Lie-Banach subgroup of $G L(\mathcal{H})$, and by Proposition [2.10, the map $\tau_{T}: G L(\mathcal{H}) \rightarrow \mathcal{E}_{n}$ is open. Therefore, the above-mentioned is the unique structure of a differentiable manifold of $\mathcal{E}_{n}$ that makes the map $\tau_{T}$ a submersion. This means that the homeomorphism of Corollary 2.11 becomes a diffeomorphism. 
Remark 2.14. A particularly interesting local cross section for $\tau_{T}$ is

$$
\sigma^{\dagger}(W)=\left(T^{\dagger} W+\left(1-T^{\dagger} T\right)\right)^{-1}=\left(T^{\dagger} W+P_{\operatorname{ker} T}\right)^{-1}
$$

defined for $W \in L(\mathcal{H}, \mathcal{K})$ such that $\|T-W\|<\left\|T^{\dagger}\right\|^{-1}$. The advantage of this section over the one defined in the proof of Proposition 2.10 is that the map

$$
(T, W) \mapsto \sigma^{\dagger}(W)
$$

is real analytic in both variables.

Theorem 2.15. Consider the map $\alpha: \mathcal{E} \rightarrow G L(\mathcal{K})^{+}$given by $\alpha(T)=T T^{*}(T \in \mathcal{E})$. Then for every $T \in \mathcal{E}$ it follows that

$$
\alpha(T \cdot G L(\mathcal{H}))=G L(\mathcal{K})^{+} .
$$

Proof. First we prove that $\left\{T A T^{*}: A \in G L(\mathcal{H})^{+}\right\}=G L(\mathcal{K})^{+}$: if $B \in G L(\mathcal{K})^{+}$, then $A=T^{\dagger} B\left(T^{\dagger}\right)^{*}+P_{\text {ker } T} \in G L(\mathcal{H})^{+}$(because $R\left(T^{\dagger}\right)=(\operatorname{ker} T)^{\perp}$ ) and it satisfies $T A T^{*}=B$. The reverse inclusion follows from Proposition 2.1

Now, $\alpha(T \cdot G L(\mathcal{H}))=\left\{T V V^{*} T^{*}: V \in G L(\mathcal{H})\right\}=\left\{T A T^{*}: A \in G L(\mathcal{H})^{+}\right\}$.

Corollary 2.16. The mapping $\alpha: \mathcal{E} \rightarrow G L(\mathcal{K})^{+}$is a splitting bundle with fibres $\alpha^{-1}(A)=A^{1 / 2} \mathcal{E}^{o}$. Moreover, for every $n \in \mathbb{N} \cup\{0, \infty\},\left.\alpha\right|_{\mathcal{E}_{n}}: \mathcal{E}_{n} \rightarrow G L(\mathcal{K})$ is a splitting bundle with global cross section $\sigma(A)=A^{1 / 2} T_{n}$, for a fixed $T_{n} \in \mathcal{E}_{n}^{o}$.

We are interested in the fibres of the bundle $\alpha_{n}: \mathcal{E}_{n} \rightarrow G L(\mathcal{K})^{+}$, given by $\alpha_{n}=\left.\alpha\right|_{\mathcal{E}_{n}}$, 1.e., $\alpha_{n}(T)=T T^{*}, T \in \mathcal{E}_{n}$. Fix $S \in G L(\mathcal{K})^{+}$and $T \in \mathcal{E}_{n}$ such that $T T^{*}=S$, i.e., $T \in \alpha_{n}^{-1}(S)$. Clearly, for every $U \in \mathcal{U}(\mathcal{H})$, also $T U^{*} \in \alpha_{n}^{-1}(S)$. Moreover:

Proposition 2.17. Let $S \in G L(\mathcal{K})^{+}$and $T \in \mathcal{E}_{n}$ such that $T T^{*}=S$. Then

$$
\alpha_{n}^{-1}(S)=T \cdot \mathcal{U}(\mathcal{H})=\left\{T U^{*}: U \in \mathcal{U}(\mathcal{H})\right\}
$$

Proof. By Corollary 2.16, $\alpha_{n}^{-1}(S)=S^{1 / 2} \mathcal{E}_{n}^{o}$. By Proposition 2.8, given $V_{1}, V_{2} \in \mathcal{E}_{n}^{o}$, there exists $U \in \mathcal{U}(\mathcal{H})$ such that $V_{1} U^{*}=V_{2}$, showing formula 2.5).

Remark 2.18. Since the fibration $\alpha_{n}: \mathcal{E}_{n} \rightarrow G L(\mathcal{K})^{+}$splits by means of the global cross section defined in Corollary 2.16, it follows that, for every fixed $T \in \mathcal{E}_{n}, \mathcal{E}_{n}$ is diffeomorphic to $G L(\mathcal{K})^{+} \times T \cdot \mathcal{U}(\mathcal{H})$. The geometry of the space $G L(\mathcal{K})^{+}$is very well studied; see [8], [6]. The study of the geometry of the fibre $T \cdot \mathcal{U}(\mathcal{H})$ (which is also an orbit) will be done elsewhere.

Splitting curves. Fix $T \in \mathcal{E}_{n}$. Recall that the space $\mathcal{E}_{n}$ is open in $L(\mathcal{H}, \mathcal{K})$ and that $\mathcal{E}_{n}=T \cdot G L(\mathcal{H})$ is the orbit of $T$ by the action of $G L(\mathcal{H})$. We shall describe now the geometry of $\mathcal{E}_{n}$. The proofs of the statements of this section appear in [8]. Denote by

$$
\mathcal{S}_{n}=\left\{(T, S) \in L(\mathcal{H}, \mathcal{K}) \times L(\mathcal{K}, \mathcal{H}): T \in \mathcal{E}_{n}, T S=I\right\} .
$$

This space has a rich geometrical structure by the action of $G L(\mathcal{H})$ given by

$$
W \cdot(T, S)=\left(T W^{-1}, W S\right), \quad W \in G L(\mathcal{H}) .
$$

In fact, for any fixed pair $(T, S) \in \mathcal{S}_{n}$, the map $\tau: G L(\mathcal{H}) \rightarrow \mathcal{S}_{n}, \tau(W)=W \cdot(T, S)$ defines a homogeneous reductive space with a connection given by the distribution of horizontal spaces. Note that the map $\tau: S_{n} \rightarrow \mathcal{E}_{n}, \tau(T, S)=T$ defines a fibre bundle with affine fibres. 
Given a smooth curve $\gamma:[0,1] \rightarrow S_{n}$ such that $\gamma(0)=(T, S), \gamma(t)=(a(t), b(t))$, the unique solution of the differential equation

$$
\left\{\begin{array}{ccc}
\dot{\Gamma} & = & \dot{a} b-a \dot{b}(1-a b) \Gamma \\
\Gamma(0) & = & I
\end{array}\right.
$$

satisfies that $\Gamma(t) \in G L(\mathcal{H}), \Gamma(t) \cdot(T, S)=\gamma(t)$. Consider now a smooth curve $\delta:[0,1] \rightarrow \mathcal{E}_{n}$, and define

$$
\gamma(t)=\left(\delta(t), \delta(t)^{\dagger}\right)=\left(\delta(t), \delta(t)^{*}\left[\delta(t) \delta(t)^{*}\right]^{-1}\right) .
$$

Observe that $\gamma(t) \in \mathcal{S}_{n} \forall t$ and $\gamma(0)=\left(\delta(0), \delta(0)^{\dagger}\right)$. Then $\delta(t)=\delta(0) \Gamma(t)^{-1}$.

Remark 2.19. In [2], R. Balan implicitly studies the following action of $G L(\mathcal{H})$ over $\mathcal{E}$ :

$$
G L(\mathcal{H}) \times \mathcal{E} \rightarrow \mathcal{E} \quad(V, T) \mapsto V T .
$$

This action is free: $V_{1} T=V_{2} T$ only if $V_{1}=V_{2}$. The orbit of $T \in \mathcal{E}$ under this action is much smaller than the orbit under the action we considered. In fact, Balan proves that $T_{1} \in \mathcal{E}$ belongs to the orbit of $T$ if and only if $R\left(T_{1}^{*} T_{1}\right)=R\left(T^{*} T\right)$. However, under the action $G L\left(\ell^{2}\right) \times \mathcal{E} \rightarrow \mathcal{E},(W, T) \mapsto T W^{-1}, T_{1} \in \mathcal{E}$ belongs to the orbit of $T$ if and only if $T_{1}^{*} T_{1}$ is congruent to $T^{*} T$, in the sense that there exists $W \in G L\left(\ell^{2}\right)$ such that $W^{*}\left(T_{1}^{*} T_{1}\right) W=T^{*} T$. Of course, this condition does not imply that $R\left(T_{1}^{*} T_{1}\right)=R\left(T^{*} T\right)$. The converse, however, is true. The relevant fact about Balan's orbits is that there is a natural metric defined on each orbit, and he uses this metric to find, given a frame $\Xi$, its closest tight frame. We shall study metrics in our orbits elsewhere.

Remark 2.20. Let $C R_{n}^{+}(\mathcal{H})$ denote the set of all positive (semidefinite) closed range operators $A$ on $\mathcal{H}$ such that $\operatorname{dim} \operatorname{ker} A=n$.

From some results obtained in [7], where the congruence orbits of any positive operator are studied, it follows that the map

$$
\beta_{n}: \mathcal{E}_{n} \rightarrow C R_{n}^{+}(\mathcal{H}), \quad \beta_{n}(T)=T^{*} T
$$

has continuous local cross sections for $n \in \mathbb{N} \cup\{0\}$. The result fails if $n=\infty$. Analogous results hold for the maps $\mu_{n}: C R_{n}^{+}(\mathcal{H}) \rightarrow C R_{n}^{+}(\mathcal{H}), \mu_{n}(A)=A^{\dagger}$ and $\theta_{n}: C R_{n}^{+}(\mathcal{H}) \rightarrow L(\mathcal{H}), \theta_{n}(A)=P_{(\operatorname{ker} A)^{\perp}}$. The fact about $\beta_{n}$ allows the study of $\mathcal{E}_{n}$ as the total space of a fibre bundle over $C R_{n}^{+}(\mathcal{H})$. It should be mentioned that the geometry of $C R_{n}^{+}(\mathcal{H})$ is well known [7], so that the fibration properties of $\beta_{n}$ may be of great help in order to completely understand the geometry of $\mathcal{E}_{n}$.

\section{Frames}

Consider a sequence $\Xi=\left(\xi_{n}\right)_{n \in \mathbb{N}}$ in a Hilbert space $\mathcal{H}$; $\Xi$ is called a Bessel sequence if there exists a positive number $B$ such that

$$
\sum_{n=1}^{\infty}\left|\left\langle\xi, \xi_{n}\right\rangle\right|^{2} \leq B\|\xi\|^{2}, \quad \xi \in \mathcal{H} .
$$

Proposition 3.1. For a sequence $\Xi=\left(\xi_{n}\right)$ in a Hilbert space $\mathcal{H}$, the following are equivalent:

(1) $\Xi$ is a Bessel sequence;

(2) there is a bounded linear operator $W: \mathcal{H} \rightarrow \ell^{2}$ such that

$$
W \xi=\left(\left\langle\xi, \xi_{n}\right\rangle\right)_{n \in \mathbb{N}}, \quad \xi \in \mathcal{H}
$$


(3) there is a bounded linear operator $T: \ell^{2} \rightarrow \mathcal{K}$ such that $T e_{n}=\xi_{n}$, where $e_{n}$ denotes the $n$-th vector of the canonical orthonormal basis of $\ell^{2}$.

The proof is straightforward. Observe that, if $\Xi$ is a Bessel sequence, then $\|W \xi\|_{2} \leq B^{1 / 2}\|\xi\|_{\mathcal{H}}$ for all $\xi \in \mathcal{H}$. In this case, $T=W^{*}$. As a corollary, the set $\mathcal{B}_{\mathcal{H}}$ of Bessel sequences in $\mathcal{H}$ is a $\mathbb{C}$-vector space. Moreover, if we define

$$
\begin{aligned}
\|\Xi\|_{\mathcal{B}} & =\inf \left\{B^{1 / 2}: \sum\left|\left\langle\xi, \xi_{n}\right\rangle\right|^{2} \leq B\|\xi\|^{2}, \xi \in \mathcal{H}\right\} \\
& =\sup \left\{\left(\sum\left|\left\langle\xi, \xi_{n}\right\rangle\right|^{2}\right)^{1 / 2}: \xi \in \mathcal{H},\|\xi\| \leq 1\right\} \\
& =\sup \left\{\left\|\sum_{n=1}^{\infty} a_{n} \xi_{n}\right\|:\left(a_{n}\right) \in \ell^{2},\left\|\left(a_{n}\right)\right\|_{2}=1\right\}
\end{aligned}
$$

then $\left(\mathcal{B}_{\mathcal{H}},\|\cdot\|_{\mathcal{B}}\right)$ is a Banach space isometrically isomorphic to $L\left(\ell^{2}, \mathcal{H}\right)$ : the isomorphism maps a Bessel sequence $\Xi=\left(\xi_{n}\right)$ into the operator $T_{\Xi} \in L\left(\ell^{2}, \mathcal{H}\right)$ defined by

$$
T_{\Xi}\left(\left(a_{n}\right)_{n \in \mathbb{N}}\right)=\sum_{n=1}^{\infty} a_{n} \xi_{n}, \quad\left(a_{n}\right)_{n \in \mathbb{N}} \in \ell^{2} ;
$$

the inverse isomorphism maps $T \in L\left(\ell^{2}, \mathcal{H}\right)$ into $\Xi_{T}=\left(T e_{n}\right)$. Analogously, we have a (conjugate-linear) isomorphism between $\mathcal{B}_{\mathcal{H}}$ and $L\left(\mathcal{H}, \ell^{2}\right)$ given by $\mathcal{B}_{\mathcal{H}} \ni \Xi \mapsto W_{\Xi}$, where $W_{\Xi}(\xi)=\left(\left\langle\xi, \xi_{n}\right\rangle\right)_{n \in \mathbb{N}}, \xi \in \mathcal{H}$, i.e., $W_{\Xi}=T_{\Xi}{ }^{*}$.

A Bessel sequence $\Xi=\left(\xi_{n}\right)$ is called a frame in $\mathcal{H}$ if there exist positive constants $A, B$ such that

$$
A\|\xi\|^{2} \leq \sum\left|\left\langle\xi, \xi_{n}\right\rangle\right|^{2} \leq B\|\xi\|^{2}, \quad \xi \in \mathcal{H} .
$$

Observe that this condition together with equation (3.1) is equivalent to

$$
A\langle\xi, \xi\rangle \leq\left\langle T_{\Xi} T_{\Xi}{ }^{*} \xi, \xi\right\rangle \leq B\langle\xi, \xi\rangle, \xi \in \mathcal{H},
$$

or, what is the same,

$$
A I_{\mathcal{H}} \leq T_{\Xi} T_{\Xi}{ }^{*} \leq B I_{\mathcal{H}} .
$$

Of course, this means that $T_{\Xi}$ is an epimorphism from $\ell^{2}$ onto $\mathcal{H}$ or, equivalently, that $W_{\Xi}=T_{\Xi}{ }^{*} \in L\left(\mathcal{H}, \ell^{2}\right)$ is bounded from below. Thus the isomorphism $\Theta$ : $L\left(\ell^{2}, \mathcal{H}\right) \rightarrow \mathcal{B}_{\mathcal{H}}, \Theta(T)=\left(T e_{n}\right)$ maps the set of epimorphisms in $L\left(\ell^{2}, \mathcal{H}\right)$ onto the set $\mathcal{F}_{\mathcal{H}}$ of all frames in $\mathcal{H}$. Observe that the positive invertible $T_{\Xi} T_{\Xi}{ }^{*} \in L(\mathcal{H})$ is usually called the frame operator of $\Xi$. $T_{\Xi}$ is called the synthesis operator of $\Xi$, and $W_{\Xi}=T_{\Xi}{ }^{*}$ is called the analysis operator of $\Xi$ ([19], [4).

A frame $\Xi=\left(\xi_{n}\right)$ is called tight if there exists $A>0$ such that

$$
\sum\left|\left\langle\xi, \xi_{n}\right\rangle\right|^{2}=A\|\xi\|^{2}, \quad \xi \in \mathcal{H} .
$$

This means that $T_{\Xi} T_{\Xi}{ }^{*}=A I_{\mathcal{H}}$, so that the set $\mathcal{F}_{\mathcal{H}}^{o}$ of tight frames in $\mathcal{H}$ corresponds (under the isomorphism $\Theta$ ) with the set $\mathbb{R}^{+} \mathcal{E}_{0}$ of positive scalar multiples of coisometries from $\mathcal{H}$ into $\ell^{2}$.

A frame $\Xi=\left(\xi_{n}\right)$ is called exact if no proper subsequence of $\Xi$ is a frame. It is known ([20], [13] ) that this is equivalent to $\left(\xi_{n}\right)$ being a Riesz basis or, what is the same, to $T_{\Xi}$ (or $W_{\Xi}$ ) being invertible.

There is a natural action of $G L\left(\ell^{2}\right)$ over $\mathcal{F}_{\mathcal{H}}$. In fact, given $\Xi \in \mathcal{F}_{\mathcal{H}}$ and $V \in$ $G L\left(\ell^{2}\right)$, define $V \cdot \Xi=\left(\left(T_{\Xi} \circ V^{-1}\right) e_{n}\right)$. In terms of the matrix $A=\left(a_{n m}\right)$, where 
$a_{n m}=\left\langle V^{-1} e_{n}, e_{m}\right\rangle, V \cdot \Xi$ is defined as the (formal) product $A \Xi$, i.e., $V \cdot \Xi=\left(\eta_{n}\right)$ where

$$
\eta_{n}=\sum_{m=1}^{\infty} a_{n m} \xi_{m}
$$

This action corresponds bijectively with that of $G L\left(\ell^{2}\right)$ over $\mathcal{E}$, so that the orbits are the connected components of $\mathcal{F}_{\mathcal{H}}$. The next result collects similar facts on $\mathcal{F}=\mathcal{F}_{\mathcal{H}}$ to those proved for $\mathcal{E}$. The proof follows from the fact that $\Theta$ is an isomorphism.

Theorem 3.2. Let $\mathcal{H}$ be a (separable) Hilbert space.

(1) $\mathcal{F}$ is an open subset of $\mathcal{B}_{\mathcal{H}}$, so that the connected components are arcwise connected.

(2) Any connected component of $\mathcal{F}_{\mathcal{H}}$ has the form

$$
\mathcal{F}_{n}=\left\{\Xi \in \mathcal{F}: \operatorname{dim} \operatorname{ker} T_{\Xi}=n\right\}
$$

for $n \in \mathbb{N} \cup\{\infty\}$. In particular, the set of Riesz basis elements of $\mathcal{H}$ is arcwise connected.

(3) If $\Xi=\left(\xi_{n}\right) \in \mathcal{F}_{n}$, then any other $E=\left(\eta_{n}\right) \in \mathcal{F}_{n}$ has the form $E=V \cdot \Xi$ for some $V \in G L\left(\ell^{2}\right)$.

(4) For any $\Xi \in \mathcal{F}_{n}$ the map $G L\left(\ell^{2}\right) \rightarrow \mathcal{F}_{n}$, defined by $V \mapsto V \cdot \Xi$, is a homogeneous space with isotropy group $G_{\Xi}=\left\{V \in G L\left(\ell^{2}\right): V \cdot \Xi=\Xi\right\}$; analogously, $\mathcal{U}\left(\ell^{2}\right) \rightarrow \mathcal{F}_{n}^{o}$, defined by $U \mapsto U \cdot \Xi$, is a homogeneous space with isotropy group $\mathcal{U}_{\Xi}=\left\{U \in \mathcal{U}\left(\ell^{2}\right): U \cdot \Xi=\Xi\right\}$.

(5) Any continuous (resp. differentiable) curve $\gamma$ in $\mathcal{F}_{n}$ such that $\gamma(0)=\Xi$ has the form $t \mapsto \Gamma(t) \cdot \Xi$ for some continuous (resp. differentiable) curve $\Gamma$ in $G L\left(\ell^{2}\right)$. Analogously, any curve $\gamma$ in $\mathcal{F}_{n}^{o}$ with $\gamma(0)=\Xi$ has the form $\gamma(t)=\Gamma(t) \cdot \Xi$ where $\Gamma$ is a curve in $\mathcal{U}(\mathcal{H})$.

(6) $\mathcal{F}_{n}^{o}$ is a deformation retract of $\mathcal{F}_{n}$ for all $n$.

Remark 3.3. Let $\Xi$ be a frame in $\mathcal{H}$. If $\Xi \in \mathcal{F}_{n}$, Balan et al [3] say that $\Xi$ has excess $n$. Then by item 2 of the last theorem, two frames can be joined by a smooth curve in $\mathcal{F}$ if and only if they have the same excess. Recall that a frame with finite excess is what Holub [16] calls a near-Riesz basis.

A Bessel sequence $\Xi$ is called a Riesz sequence if $T_{\Xi}$ is bounded from below or, equivalently, if $W_{\Xi}$ is an epimorphism. In other words, if there exist $A, B>0$ such that

$$
\forall c \in \ell^{2}, \quad A\|c\|_{2} \leq\left\|\sum_{n \in \mathbb{N}} c_{n} \xi_{n}\right\|_{\mathcal{H}} \leq B\|c\|_{2} .
$$

We shall denote by $\mathcal{R}=\mathcal{R}_{\mathcal{H}}$ the space of Riesz sequences in $\mathcal{H}$. As before we denote by $\mathcal{R}_{n}=\left\{\Xi \in \mathcal{R}: \operatorname{dim} \operatorname{ker} W_{\Xi}=n\right\}, n \in \mathbb{N} \cup\{\infty\}$. In the terminology of Balan et al [3], the number $n=\operatorname{dim} \operatorname{ker} W_{\Xi}$ is called the deficit of the sequence $\Xi$.

Using the (conjugate linear) isomorphism between $\mathcal{B}_{\mathcal{H}}$ and $L\left(\mathcal{H}, \ell^{2}\right)$, which maps $\mathcal{R}$ onto $\mathcal{E}\left(\mathcal{H}, \ell^{2}\right)$, we can make a similar analysis for Riesz sequences as we made for frames. In this case the action of the group $G L(\mathcal{H})$ on $\mathcal{R}$ is given by $V \cdot \Xi=$ $\left(\left(V^{-1}\right)^{*} \xi_{n}\right)_{n \in \mathbb{N}}$ for $\Xi=\left(\xi_{n}\right)_{n \in \mathbb{N}} \in \mathcal{R}$.

As in the case of frames, one can get a classification of Riesz sequences by translating the classification of epimorphisms done in section 2. Thus, $\mathcal{R}_{\mathcal{H}}$ is an open subset of $\mathcal{B}_{\mathcal{H}}$, whose connected components are the sets $\mathcal{R}_{n}, n \in \mathbb{N} \cup\{\infty\}$, which are also the orbits of the action of $G L(\mathcal{H})$ on $\mathcal{R}$. These orbits are homogeneous spaces 
of $G L(\mathcal{H})$. The isotropy group of a fixed $\Xi \in \mathcal{R}_{n}$ is given by those $V \in G L(\mathcal{H})$ such that $V^{*}$ acts as the identity on the closure of the subspace generated by $\Xi$.

Note that the subset of $\mathcal{R}$ associated to the co-isometries $\mathcal{E}^{0}\left(\mathcal{H}, \ell^{2}\right)$ coincides with the set of orthonormal systems in $\mathcal{H}$. Then, for every fixed $n \in \mathbb{N} \cup\{\infty\}$, the set of orthonormal systems with deficit $n$ is a deformation retract of the orbit $\mathcal{R}_{n}$.

Remark 3.4. Another way to get the previous analysis for $\mathcal{R}_{\mathcal{H}}$ consists in translating the geometry of $\mathcal{E}\left(\mathcal{H}, \ell^{2}\right)$ to the subset of $L\left(\ell^{2}, \mathcal{H}\right)$ of those operators that are bounded from below, using the diffeomorphism $W \mapsto W^{*}$. Then the Riesz sequences can be classified by identifying them with their synthesis operators (instead of using the analysis operators as we did before).

Remark 3.5. The separability hypothesis is not an essential one. In fact, all results can be proven in the general sense, using minor changes. On the other hand, the results of the paper can easily be generalized to the setting of frames in Hilbert $\mathrm{C}^{*}$-modules.

\section{ACKNOWLEDGMENT}

We thank the referee for several useful comments which helped us to improve the paper. In particular, the addition of the classification of the Riesz sequences is due to a suggestion of the referee, who also informed us about the recent paper [3].

\section{REFERENCES}

1. Aldroubi, Akram; Portraits of frames, Proc. Amer. Math. Soc. 123 (1995), 1661-1668. MR 95g:46037

2. Balan, Radu; Equivalence relations and distances between Hilbert frames, Proc. Amer. Math. Soc. 127 (1999), 2353-2366. MR 99j:46025

3. Balan, R., Casazza, P. G., Heil, C. and Landau, Z.; Deficits and excesses of frames, Adv. in Computational Math. 18 (2003), 93-116. MR 2004a:42040

4. Casazza, Peter G; The art of frame theory, Taiwanese J. Math. 4 (2000), 129-201. MR 2001f: 42046

5. Christensen, Ole; Frames, Riesz bases, and discrete Gabor/wavelet expansions, Bull. Amer. Math. Soc. (N.S.) 38 (2001), 273-291. MR 2002c:42040

6. Corach, G. and Maestripieri, A.; Differential and metrical structure of positive operators, Positivity 3 (1999), 297-315. MR 2001a:58004

7. Corach, G., Maestripieri, A. and Stojanoff, D.; Orbits of positive operators from a differentiable viewpoint, preprint.

8. Corach, G., Porta, H. and Recht, L.; Differential geometry of spaces of relatively regular operators, Integral Equations and Operator Theory 13 (1990), 771-794. MR 93a:46099

9. Cordes, H. O. and Labrousse, J. P.; The invariance of the index in the metric space of closed operators, J. Math. Mech. 12 (1963) 693-719. MR 28:5341

10. Daubechies, I.; Ten lectures on wavelets, CBMS-NSF Regional Conference Series in Applied Mathematics 61, Society for Industrial and Applied Mathematics (SIAM), Philadelphia, PA, 1992. MR 93e:42045

11. Daubechies, I., Grossmann, A. and Meyer, Y.; Painless nonorthogonal expansions, J. Math. Phys. 27 (1986), 1271-1283. MR 87e:81089

12. Desoer, C. A. and Whalen, B. H.; A note on pseudoinverses, J. Soc. Indust. Appl. Math. 11 (1963), 442-447. MR 27:6128

13. Duffin, R. J. and Schaeffer, A. C.; A class of nonharmonic Fourier series, Trans. Amer. Math. Soc. 72 (1952), 341-366. MR 13:839a

14. Han, Deguang and Larson, David R.; Frames, bases and group representations, Mem. Amer. Math. Soc. 147 (2000). MR 2001a:47013

15. Heil, Christopher E. and Walnut, David F.; Continuous and discrete wavelet transforms, SIAM Rev. 31 (1989), 628-666. MR 91c:42032 
16. Holub, J. R.; Pre-frame operators, Besselian frames, and near-Riesz bases in Hilbert spaces, Proc. Amer. Math. Soc. 122 (1994), 779-785. MR 95a:46030

17. Munkres, J. R.; Topology: a first course, Prentice-Hall, Englewood Cliffs, New Jersey, 1975. MR 57:4063

18. Nashed, M. Z.; Generalized inverses and applications, Academic Press, New York, 1976. MR 56:9943

19. Ron, A. and Shen, Z.; Affine systems in $L_{2}\left(\mathbf{R}^{d}\right)$ : the analysis of the analysis operator, J. Funct. Anal. 148 (1997), 408-447. MR 99g:42043

20. Young, Robert M.; An introduction to nonharmonic Fourier series, revised first edition, Academic Press, San Diego, CA, 2001. MR 2002b:42001

Depto. de Matemática, Facultad de Ingeniería UBA, Buenos Aires (1063), Argentina

E-mail address: gcorach@fi.uba.ar

Depto. de Matemática, Facultad de Ingeniería, UnPSJB, C. Rivadavia (9000), ArGENTINA

E-mail address: mep@unpata.edu.ar

Depto. de Matemática, FCE-UnlP, La Plata (1900), Argentina

E-mail address: demetrio@mate.unlp.edu.ar 